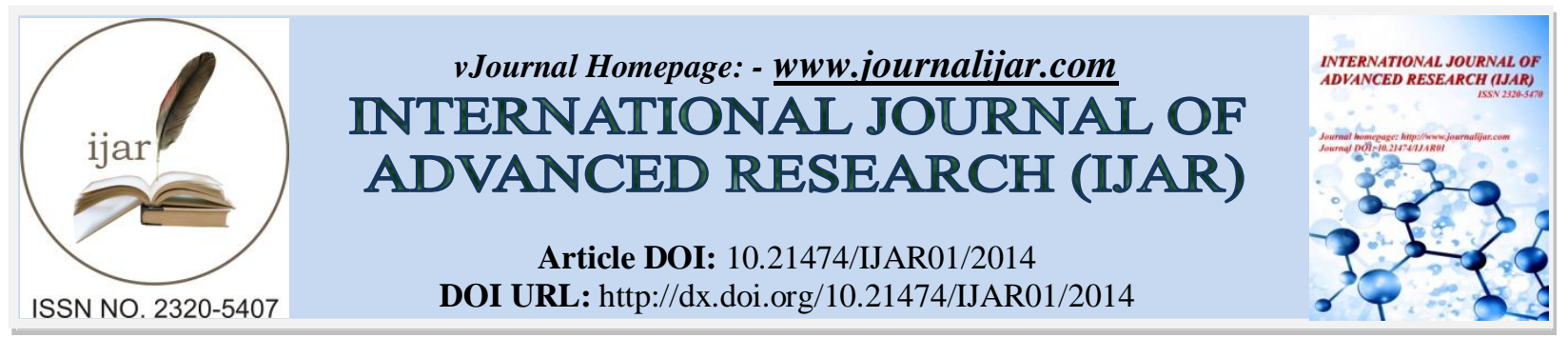

RESEARCH ARTICLE

\title{
STUDY OF QUANTIZED CURRENT IN NANO ELECTRICAL CIRCUITS WITH DIFFERENT CONFIGURATIONS OF CELLS.
}

Ashrafuz Zaman Sk. (M.Sc, B.Ed,SLET) and Dr.Ranjit Choudhury. Assistant professor Science Academy, Goalpara.

\section{Manuscript Info}

Manuscript History

Received: 22 September2016

Final Accepted: 6 October 2016

Published: October 2016

Key words:-

Quantized, Potential well, Quantum state, Emf, Ohms law ,Configuration,

\section{Abstract}

The field of carbon nano tubes (CNT) is an active and promising area of research theoretically as well as experimentally[1],[2]. In fact, the band structure of CNT determines its conductivity and in carbon nano tubes the structural pattern affects the conductivity type .It is well established Phenomenon that samples of single wall carbon nano tubes with the arm chair wrapping have been produced and exhibit metallic behaviour. In this paper we consider the carbon nano resistors which are in fact conductors. There fore, the well established quantized resistance of the conducting carbon nano resistors is taken as the starting point, from which we try to find the quantized current of electrons with the help of Ohm's law in various nano circuits containing several sources of E.M.F in series, parallel and mixed grouped configurations provided those circuits comprise of several carbon nano resistors(wires).Finally, in mixed group of configuration maximum current through the circuit is determined in aid of Ohm's law.

Copy Right, IJAR, 2016,. All rights reserved.

\section{Introduction:-}

From the point of view of the theoretical Physics ,the assumption of a single wall one dimensional carbon nano tubes(wires) having extremely small thickness, may be considered to be the fruitful starting point for further study and for the investigation of the quntized electrical behaviour of carbon nano tubes[2], [3] even in composite structure of multiwall carbon nano tubes with several layers. .Also, it has been already established see for instance [1], when CNT is extremely narrow (thin) for a very thin conductor $\mathrm{A}^{\frac{1}{2}}<<\mathrm{L}$ where $\mathrm{A}$ is the area of cross section and $\mathrm{L}$ is the length of the potential well, the total energy of a single electron in side a one dimensional well of infinite depth is given by, $E_{n}=\frac{n^{2} \pi^{2} h^{2}}{2 m L^{2}}$ (i) ' $m$ ' is the mass of the electron.

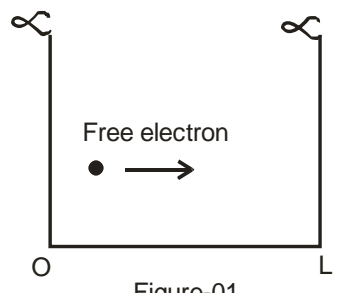

Figure-01 
For one electron system, electron spatial density

$N_{n}=\frac{1}{A L}$.

Many experiments show that CNT are ballistic conductor [2], and therefore velocity of the electron in the $n$th quantum state $v_{n}=\frac{n h}{2 m L}$.

Introducing transit time of the electron in nano tube

$\tau=\int_{O}^{L} \frac{d x}{v_{n}}=\frac{L}{v_{n}}$

$\tau_{n}=2 m \frac{L^{2}}{n h}$

With the help of (i), (ii), (iii) and (iv) we could readily establish the relation for conductivity for CNT.

$\sigma_{n}=\frac{2 L e^{2}}{A n h} \quad$ or

$\rho_{n}=\frac{A n h}{2 L e^{2}}$

Thus, quantized resistance of the conducting wire or of the is given by

$R_{n}=\rho_{n} \frac{L}{A}=\frac{n h}{2 e^{2}}$

This resistance is purely quantum in nature and state dependent, this implies $R_{n}$ is temperature dependent, see for instance [4] and[5]

\section{Method and model:-}

For dealing with our problem we consider previous model of CNT with the one dimensional approach of carbon nano tubes in which electron is confined with in the well of infinite depth, so that electron is completely free to to move with in the well. That means electron is confined from transverse directions in the tube that resuls in the quantized nature of the total energy of the electron[1].Hence one dimensional box model with single electron constitute the starting point for fruitful approach to find the quantized resistance.Apart from this, we use well established Ohm's law in order to find the quantized current through the circuit in different configuration such as series, parallel and and mixed grouping etc. There is no restriction on application of Ohm's law as so far we have assumed carbon nano resistors are conductors ..Therefore, small variation of applied voltage and current would bring no appreciable change in resistance as far as validity of Ohm's law is concerned.

\section{Theory:-}

\section{Series Configuration:-}

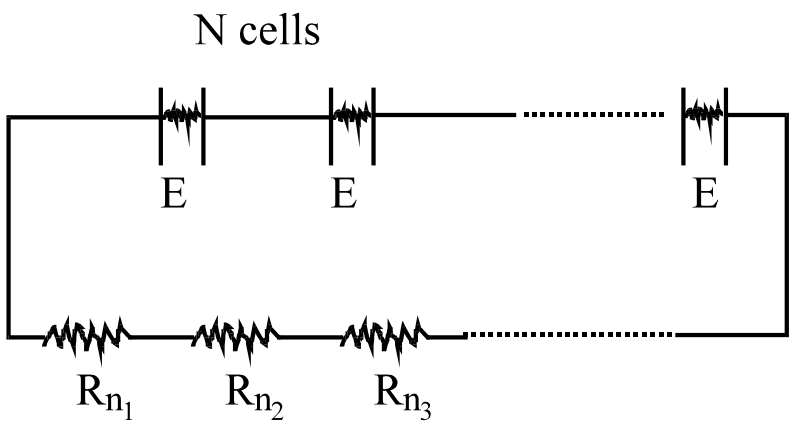

Fig-02

Let us consider a combinations of ' $N$ ' number power sources (cells) each of E.m.f ' $E$ ', all are placed in series with carbon nano resistors(carbon quantum resistor) of resistances $\boldsymbol{R}_{\boldsymbol{n}_{1},} \boldsymbol{R}_{\boldsymbol{n}_{2},} \boldsymbol{R}_{\boldsymbol{n}_{3}} \ldots \ldots \ldots$; which are also in series configuration. In practice, every cell has some internal resistance what so ever it smaller is .Let us consider every cell is bestowed with same internal resistance ' $\boldsymbol{r}$ '. Since, we are dealing with conducting nano resistors in the circuit 
we may safely apply $\mathbf{O h m}$ 's law to quantify the current through the circuit given by- $\mathbf{I}=\frac{\boldsymbol{E}}{\boldsymbol{R}_{\boldsymbol{n}}+\boldsymbol{R}_{\boldsymbol{i}}}$

$$
\mathrm{I}=\frac{N E}{R_{n}+N r}
$$

Where ' $R_{n}$ ' is the total external resistance due all the carbon nano resistors .But,. total external resistance in the nano circuit ' $\boldsymbol{R}_{\boldsymbol{n}}=\boldsymbol{R}_{\boldsymbol{n}_{1},}+\boldsymbol{R}_{\boldsymbol{n}_{2},}+\boldsymbol{R}_{n_{3}}+\cdots \ldots \ldots$.

We first consider dissimilar quantized states of all the external carbon nano resistors corresponding to states

$n_{1} \quad n_{2}, n_{3 \ldots \ldots . . e t c .} \quad$ Then, $\quad \mathrm{I}=\frac{\mathrm{NE}}{\frac{\mathrm{h}}{2 \mathrm{e}^{2}}\left(\mathrm{n}_{1}+\mathrm{n}_{2+\ldots . .}\right)+\mathrm{Nr}}$

But, if we consider that the net internal resistance due to all the cells far exceeds the net external resistance then $\frac{\mathbf{h}}{2 \mathbf{e}^{2}}\left(\mathbf{n}_{\mathbf{1}}+\mathbf{n}_{\mathbf{2}+\cdots \ldots}\right) \ll \mathbf{N r}$, In such a case current through the circuit may be given as-

$$
\begin{aligned}
\mathrm{I} \approx & \frac{N E}{N r} \\
\quad \mathrm{I} & \approx \frac{E}{r} \ldots \ldots \ldots \ldots \text { (ii) }
\end{aligned}
$$

Thus, current is in thre circuit as if there is only one cell in the arrangement. Moreover, we can see current is not prominently quantized or not quantized if we go on ignoring very negligible external resistance as compared to the internal resistance. One might think a bit of change in temperature may bring change in external resistance but infact this change is masked by a large internal resistance of the cells as we have considered, $\frac{\mathbf{h}}{2 \mathbf{e}^{2}}\left(\mathbf{n}_{1}+\mathbf{n}_{2+\cdots \ldots . . .}\right) \ll$ Nr. Quantum nature is not prominent in such an arrangement. Thus, the current through the circuit cannot be increased and current is independent of the number of cells. .Now, let us bounce back to the case when the external resistances far exceeds the total internal resistance. Thus,

$$
\frac{\mathbf{h}}{2 \mathbf{e}^{2}}\left(\mathbf{n}_{1}+\mathbf{n}_{2+\cdots \ldots}\right) \gg \mathbf{N r}
$$

Then, we can have the current in the nano circuit , that is given by- $\boldsymbol{I}_{\boldsymbol{n}}=\frac{\mathbf{N E}}{\frac{\mathrm{h}}{2 \mathrm{e}^{2}},\left(\mathbf{n}_{1}+\mathbf{n}_{2}+\ldots . . . . . . . . .(i i i)\right.}$

$$
\mathrm{I} \approx \frac{N E}{R_{n}}
$$

Thus,

the effective current of the nano circuit is quantized, also the current through the circuit is $N$ times than that of the current obtained from a single cell with external resistance $R_{n}$

If we have all the resistors having same internal structure and property, then we may assume identical states of all the carbon quantum resistors, say ' $n$ ', is the quantum state corresponding to the a certain temperature for all the carbon nano resistors, assuming there are $N$ number of such resistors then $\mathbf{I}=\frac{\mathbf{N E}}{\frac{\mathbf{h}}{2 \mathrm{e}^{2},(\mathbf{N n})}}$

$$
I_{n}=2 e^{2} \frac{E}{n h}
$$

Thus, we have seen when the numbers of external carbon resistors is same as the number of cells in series providing net external resistance far exceeds the total internal resistance, then the circuit behaves as if we have applied one single cell of E.m.f 'E with merely a single carbon nano resistor of resistance' $\frac{n h}{2 e^{2}}$

\section{Parallel configuratio:-}

Let us now consider the' $\mathrm{N}$ number of'cells being arranged in parallel configuration with the external carrbon nano resistors as shown in figure (02.) Then, the current through the nano tubes can be given as $\mathrm{I}=\frac{\boldsymbol{E}}{\boldsymbol{R}_{\boldsymbol{n}}+\boldsymbol{R}_{\boldsymbol{i}}}=\frac{\boldsymbol{E}}{\boldsymbol{R}_{\boldsymbol{n}}+\frac{\boldsymbol{r}}{\boldsymbol{N}}}$ 


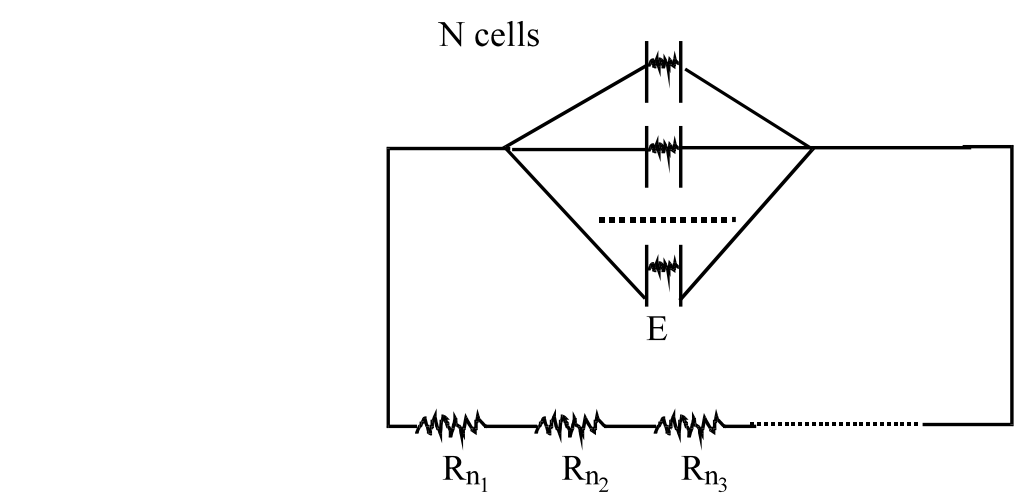

$$
\mathrm{I}=\frac{N E}{N \frac{\mathrm{h}}{2 \mathrm{e}^{2}},\left(\mathrm{n}_{1}+\mathbf{n}_{2+\cdots . . .)}\right)+r}
$$

Fig-03

Now, we consider the case when $\boldsymbol{R}_{\boldsymbol{n}} \ll \frac{r}{N}$, then $\quad \boldsymbol{N} \frac{\mathbf{h}}{2 \mathrm{e}^{2}}\left(\mathbf{n}_{1}+\mathbf{n}_{2+\cdots . . .}\right) \ll \mathbf{r}$,

Thus, current is in the circuit $\mathbf{I}=\frac{N E}{r} \ldots \ldots \ldots . . . . .$. (vi) Thus, current is in the circuit is ' $\mathbf{N}$ ' times the current obtained by a single cell of internal resistance' r' and found to be weakly quantized, i.e quantization effect is masked by the larger internal resistance or approximately speaking ,current is not quantized.

Now, we take the case when external resistances due to the carbon nano resistors far exceeds the total inter resistance of the cells. In such a case $\boldsymbol{R}_{n} \gg \frac{r}{N}$ Therefore current. through the circuit may be given by

$$
\mathbf{I}=\frac{\boldsymbol{E}}{\frac{\mathbf{h}}{2 \mathrm{e}^{2}},\left(\mathbf{n}_{1}+\mathbf{n}_{2}+\cdots \ldots\right)}
$$

Thus , the current through the circuit is as if there is only one cell with external resistance $\mathbf{R}_{\mathbf{n}}$. Now , if we are free to replace the all the carbon nano resistors by a single carbon resistor of equivalent quantum state ' $\mathbf{n}$ "*, without affecting the current in the circuit then, we can write

$\frac{2 e^{2} E}{{ }^{*} * * h}$

\section{Mixedgrouping \\ Configuration}

We now design a nano circuit with mixed grouping of cells which includes both series and parallel connections of cells as shown in figure-( 03).In thi case too, we consider each cell of internal resistance ' $r$ '.Point to be noted, we could use single external resistor instead of taking several carbon resistors of different quantum states, this would yield the same result just replacing several quantum states by equivalent one quantum state. We denote this equivalent quantum state by $n^{* *}$ replacing all other states of several carbon nano resistors.

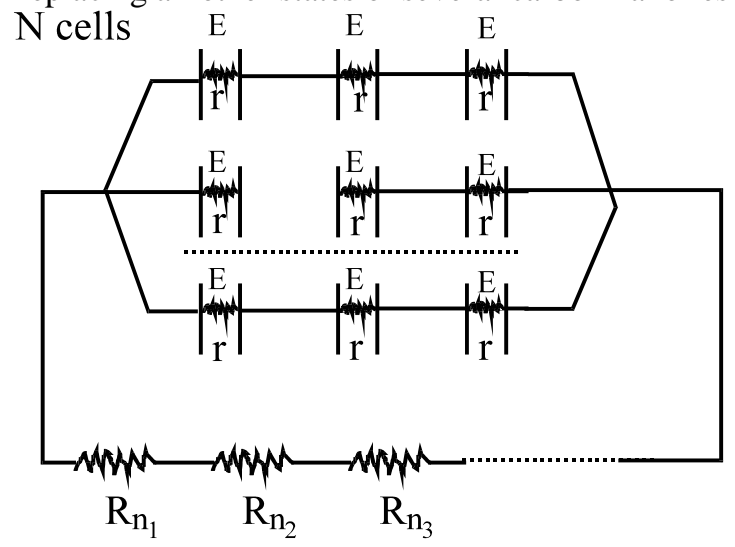

Fig-03 
Suppose, we have total $\mathbf{N}$ cells being distributed with ' $\boldsymbol{n}$ ' cells in each of ' $\mathrm{m}$ ' number of rows , arranged in mixed grouping as shown in fiure(03), then the net internal resistance in the entire arrangement is given by $\boldsymbol{R}_{\boldsymbol{i}}=\frac{\boldsymbol{n}^{\prime} \boldsymbol{r}}{\boldsymbol{m}}$

Therefore, Current in the nano circuit is given by

$$
\begin{gathered}
\mathrm{I}=\frac{n^{\prime} E}{R_{n}+\frac{n^{\prime} r}{m}} \\
\mathrm{I}=\frac{m n^{\prime} E}{m R_{n}+n^{\prime} r} \text { or } \mathrm{I}=\frac{N E}{m R_{n}+n^{\prime} r} \ldots \ldots \ldots . . .
\end{gathered}
$$

\section{Condition for maximum current:-}

Since ,NE is constant, hence maximum flow of quantized current is achieved when denominator is minimum ,i.e

$\boldsymbol{m} \boldsymbol{R}_{\boldsymbol{n}}+\boldsymbol{n}^{\prime} \boldsymbol{r}=\left(\sqrt{\boldsymbol{m} \boldsymbol{R}_{\boldsymbol{n}}}-\sqrt{\boldsymbol{n}^{\prime} \boldsymbol{r}}\right)^{\mathbf{2}}+\mathbf{2} \sqrt{\boldsymbol{m} \boldsymbol{R}_{\boldsymbol{n}} \boldsymbol{n}^{\prime} \boldsymbol{r}}$ Thus, the maximum current is obtained when,

$$
\begin{gathered}
R_{n}=\frac{n^{\prime} r}{m} \ldots \ldots \ldots \ldots \ldots \ldots . . .(\mathrm{ix}) \\
\text { Or } \quad \frac{h}{2 e^{2}}\left(\mathrm{n}_{1}+\mathrm{n}_{2+\ldots \ldots}\right)=\frac{n^{\prime} r}{m}
\end{gathered}
$$

$$
\text { , } m R_{n}=n^{\prime} r
$$

If all the carbon nano resistors are replaced by equivalent single carbon nano resistor of quantum state, $\boldsymbol{n}^{* *}$ then

$$
\begin{aligned}
& \frac{\boldsymbol{h}}{2 \boldsymbol{e}^{2}}, \boldsymbol{n}^{* *}=\frac{\boldsymbol{n}^{\prime} \boldsymbol{r}}{\boldsymbol{m}} \\
& \text { or } \quad n^{* *}=\frac{n^{\prime} r 2 e^{2}}{m h} \ldots \ldots \ldots \ldots(\mathrm{ix}) \quad \text { or } \quad r=\frac{m n^{* *} h}{2 n^{\prime} e^{2}} \ldots \ldots \ldots \ldots \ldots \ldots \ldots(x)
\end{aligned}
$$

Thus, internal resistance of each shell is $\frac{m}{n^{\prime}}$ times of the resistance $\left(\frac{m n^{* *} h}{n^{\prime} 2 e^{2}}\right)$ of external equivalent carbon nano resistor, subject to maximum current condition. Further, if there are exactly as many cells in each row as many the number of rows are, then $\mathbf{m}=\boldsymbol{n}^{\prime}$, clearly then we can write

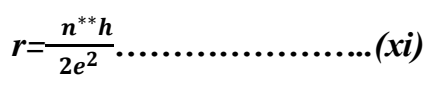

Thus most striking fact about the mixed grouping of cells is that when there are as many cells in each row as many the number of rows are , then the internal resistance of each cell should be equal to that of external equivalent resistance of the carbon nano resistor to have maximum current.

There fore the maximum current in the circuit is given by:-

$I=\frac{N E}{2 \sqrt{m R_{n} n^{\prime} r}}=\frac{N E}{2 \sqrt{m R_{n} \cdot m R_{n}}}=\frac{N E}{2 m R_{n}}$

Now ,substituting the quantized value of the external resistance in the above, we have at once,

$\mathrm{I}=\frac{N E \times 2 e^{2}}{2 m n^{* *} h}$

$\mathrm{I}=\frac{N E e^{2}}{m n^{* *} h}$ or $\quad I=\frac{n^{\prime} E e^{2}}{n^{* *} h}$,

Note that $n$ ' is the number of cells in each row.

It is now obvious that the maximum current in the nano circu it in mixed configuration of the cells varies directly with the number of cells in each row or the total number of cells but varies inversely as the quantum state of the external carbon nano resistor . 


\section{Findings of the Results:-}

My approach was totally theoretical supported by experimental back ground of quantized resistance as cited in ref.[1]. It is notable that quantized electrical behavior is prominent when we ignore the the total internal resistance of the cells. Apart from this my findings can be summarized as follows.

1. Thus, the current through the circuit cannot be increased and current is independent of the number of cells when we assumed the total internal resistance of the cells far exceeds the external resistance due to carbon nano resistors. Thus, current through the circuit is as if due to one single cell.

2. How ever if the external of resistances of the carbon nano resistors far exceed the total internal resistance of the cells, current is $\mathrm{N}$ (number of cells)times than that of obtained from a single cell.

3. Therefore, if the external of resistances of the carbon nano resistors far exceed the total internal resistance of the cells, then we must prefer series configuration of the cells. Otherwise, parallel configuration is desirable if the total internal resistance of the cells far exceeds the external resistance due to carbon nano resistors

4. we have seen when the numbers of external carbon resistors is same as the number of cells in series providing net external resistance far exceeds the total internal resistance, then the circuit behaves as if we have applied one single cell of E.m.f ' $E$ with merely a single carbon nano resistor of resistance' $\frac{\mathrm{nh}}{2 \mathrm{e}^{2}}$

5. In mixed group configuration of cells ,most striking fact is that when there are as many cells in each row as many the number of rows are, then the internal resistance of each cell cells, should be equal to that of external equivalent resistance of the carbon nano tubes

6. It is now obvious that the maximum current in the nano circuit in mixed configuration of the cells varies directly with the number cells in each row or the total number of cells but varies inversely as the effective quantum state of the external carbon nano resistor

\section{Conclusions and Remarks;-}

We assumed electron in CNT or one dimensional quantum nano resistor has a few restrictions such as $\sqrt{\mathrm{A}}<<\mathrm{L}$ and the potential well is infinite depth to have quantize nature of resistance and which in turn quantize the current in discrete values. Apart from this, in practice the internal resistance of the cells in the nano scale set up, is expected to be much more smaller than external quantized reistance of the carbon nano resistors in normal room temperature because $\frac{n h}{2 e^{2}} \approx 10^{4}$ and no internal resistance is expected to be of the order of $10^{4}$. This is in turn expresses that external resistances in the nano circuit in room temperature must exceed the internal resistance of the cells , hence current is said to be state dependent and strictly speaking current is strongly quantized in the nano circuit comprising of one dimensional carbon nano resistors .

\section{Acknowledgement}

I would like to remember and thank, my one of the most respected sir, Professor Dr. Sayed Abu Saleh Ahmed, Gauhati University for inspiring and driving my attentions towards carbon nano world for whom I am carrying on my works in this area to understand a bit the surprising world of carbon nano tubes. At the same time, I would like to acknowledge the support and help made whenever required by Professor Dr Ranjit choudury, Goalpara College, Goalpara. I feel so happy to have such resourceful persons as my inspiration for developing my inherent interest in Physics one way and the other way to inspire me for exaltation and improvement as a human.

\section{References:-}

1. CAFFARO MAGRADO \& -CAFFARO M. GRADO (2001) Quantization of the electrical conductivity in CNT.

2. Boxiao chen. (PH-464 Applied optics), Instructor-Andress Larose. Quantized electrical conductance of carbon nano tubes

3. Sk Zaman Ashrfuz, Chowdhury, Dr. Ranjit (2016) Non dependence of Drift velocity of Electron on length of carbon nano tubes, Dependence on Quantum state and Expression of current density -

4. Sk Zaman Ashrfuz, Chowdhury, Dr. Ranjit (2016)Temperature dependendence Quantum state Of Electron in One Dimensional Carbon nano tube and the expression for Temperature coefficient of Resistance in terms of Quantum State

5. MELE E. J., LEE L.S., FISCHER JE, H. DAI, AHESS, RE SMALLEY. Etc. (1998) Temperature depended resistivity of single well of carbon nano tubes

6. Gupta Satish.k,(2005)Modern abc of Physics-VOL-II FIFTEENTH EDITION Modren publishe189,190 